\title{
Theoretical gas phase compressibility factor of mixed refrigerants in auto-cascade refrigeration system
}

\author{
Zheng Da-yu(a) ${ }^{(a)}$ B. Liu-Lei ${ }^{(b)}$, B. Gao Li-ping ${ }^{(b)}$, C. Chen Qiu-yan C. Chen- \\ shuo $^{(\mathrm{c})}$, D. Yu Hai-feng ${ }^{(\mathrm{d})}$, D. Li Xiang ${ }^{(\mathrm{d})}$, D. Li Jie ${ }^{(\mathrm{d})}$ \\ (a) School of Energy and Civil Engineering of Harbin Commercial University \\ Harbin, 150028, China \\ Fax086-451-84865269, zhengdayu2000@126.com \\ (b) School of Energy and Civil Engineering of Harbin Commercial University \\ Harbin, 150028, China
}

Fax086-451-84865269, superteacher73@126.com

\begin{abstract}
KEYWORD: auto -cascade; mixed refrigerants; gas phase compressibility factor; ratio of refrigerant ABSTRACT: The compressibility factor is solved using the RKS equation and the corresponding calculation program. Redlich-kwong- Soave the numerical calculation method for solving the compressibility factor, $\mathrm{R} 22$ and $\mathrm{CO} 2$ is calculated and compare it with standard values, so as to verify correctness of program. Finally, calculate the compressibility factor of mixed refrigerants R14/R23/R22 in a three-stage auto cascade system. At the same time, provide reference for solving it in auto-cascade system.
\end{abstract}

\section{INTRODUCTION}

Auto-cascade refrigeration system uses different boiling point of mixed refrigerants components, through a series of heat transfer, mass transfer to achieve gas-liquid separation, so as to get liquid component then achieve a low evaporation temperature with a single stage compressor. As autocascade refrigeration system need multistage separation and refrigerant in evaporator, condenser, separator is gas-liquid two phase, gas liquid phase composition affects the separation effect of gas liquid separator and refrigeration efficiency. Compression factor is a physical quantities representing the degree of real gas deviating from ideal gas, it defined as $z=\frac{P V}{R T}$.It can accurately express the relations of real gas among pressure, volume, temperature, and it can be used to calculate thermodynamic functions-fugacity, steam pressure and so on. With compression factor, the quality of gas and liquid and proportion of refrigerant can be calculated.Therefore, solving refrigerator compesssibility factor in different device is become more and more important.

Although compressibility factor of some substances is known, compressibility factor changes with the temperature. If don't consider the influence of temperature on it, then it will produce error. For now, there is no authority data to look up for mixed refrigerant. So this article aims at exploring the numerical calculation method and writing program, so as to solve compressibility factor of mixed refrigerant. With compression factor, it can be derived that quality of gas and liquid, then derive thermal physical properties of refrigerant. As a result, optimize the proportion of refrigerant, so as to improve the efficiency of the whole system.

\section{Computing process}

\section{RKS equation}

RKS equation is the most successful state equation by far, its form as follows:

$$
P=\frac{R T}{V-b}-\frac{a(T)}{V(V+b)}
$$

where $b=\Omega_{b} \frac{R T_{C}}{P_{C}}, \Omega_{b}=0.8664$;

$$
a=a_{c} \alpha(T), a_{c}=\Omega_{a} \frac{\left(R T_{C}\right)^{2}}{P_{C}}, \Omega_{a}=0.42748 ;
$$


$\alpha(\mathrm{T})$ changes with temperature, with the following

$$
\alpha(T)=1+\left(1-\frac{T}{T_{C}}\right)\left(m+n \frac{T_{C}}{T}\right)
$$

where $m$ and $n$ is constant, it depends on the nature of refrigerants.

So RKS equation is converted to:

RKS80: $\quad P=\frac{R T}{V-b}-\frac{\alpha_{c}\left\{1+L\left[1-\left(\frac{T}{T_{C}}\right)^{0.5}\right]\right\}^{2}}{V(V+b)}$

In formula (3), we can get $\mathrm{m}$ and $\mathrm{n}$ from relevant information, if the compressibility factor solved with $\mathrm{m}$ and $\mathrm{n}$ has a big error, then update the values of $\mathrm{m}$ and $\mathrm{n}$. so as to get more optimized values.

Thus, this article use RKS80 equation to calculate thermodynamic properties.

Define $A=\frac{P a}{(R T)^{2}}, B=\frac{P b}{R T}, Z=\frac{P V}{R T}$, then RKS80 equation is changed to a equation with compression factors as follows.

$Z^{3}-Z^{2}+\left(A-B-B^{2}\right) Z-A B=0$

Due to the complex form of $Z$ in equation, so the equation need be solved with iterative method. This article uses Newton iteration method, the calculation form is as follows:

$Z_{2}=Z_{1}-\frac{F\left(Z_{1}\right)}{F_{Z_{1}}\left(Z_{1}\right)}$

Where $Z_{1}, Z_{2}$ is old and new values of compression factors. $F_{Z 1}\left(Z_{1}\right)$ is $\mathrm{F}\left(Z_{1}\right)$ took derivative of $Z_{1}$.

So we can get the equation as follows:

$$
F\left(Z_{1}\right)=Z^{3}-Z^{2}+\left(A-B-B^{2}\right) Z-A B=0
$$

\section{Computing process}

Concrete steps of iteration method to solve the compression factor is as follows.

(1)The initial approximate roots $Z_{0}$ and precision $\varepsilon$ is given.

(2)Calculate $Z_{2}=Z_{1}-\frac{F\left(Z_{1}\right)}{F_{Z_{1}}\left(Z_{1}\right)}$

(3)If $\left|z_{1}-z_{2}\right|<\varepsilon$, then return to $\operatorname{step}(4)$.Otherwise $z_{0}=z_{1}$, convert to $\operatorname{step}(2)$;

(4)Output $Z_{1}$, which content with conditions of premise, end.

This article use Newton iterative method to solve the compression factor, the main flow chart is as follows:

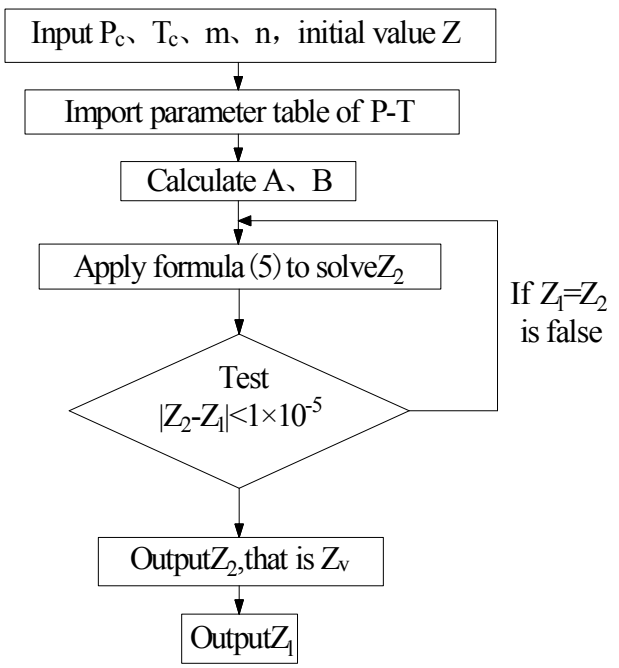

Figure 1 Newton iterative method to solve the compression factor 


\section{Program verification}

In order to testify the correctness of program, we select state points of $\mathrm{R} 22$ and $\mathrm{CO}_{2}$ in different temperature. Calculate compressibility factor of every state points with the program, then compare it with the standard values.

\section{Compressibility factor of R22}

The standard values and solved compressibility is shown as table1.

\begin{tabular}{cccc} 
Table 1. & R22 data and calculated compressibility factor \\
\hline $\mathrm{T}\left({ }^{\circ} \mathrm{C}\right)$ & $\mathrm{P}(\mathrm{Mpa})$ & $\begin{array}{c}\text { Calculation } \\
\text { value }\end{array}$ & $\begin{array}{c}\text { Standard } \\
\text { value }\end{array}$ \\
\hline- & 0.002 & 0.9985 & 0.99 \\
100 & & 1 & 0.98 \\
-60 & 0.04 & 0.9542 & 0.97 \\
-30 & 0.164 & 0.8988 & 0.91 \\
0 & 0.498 & 0.8869 & 0.89 \\
5 & 0.584 & 0.8744 & 0.87 \\
10 & 0.68 & 0.8464 & 0.83 \\
20 & 0.91 & 0.7808 & 0.77 \\
40 & 1.534 & 0.743 & 0.72 \\
50 & 1.943 & 0.6574 & 0.63 \\
70 & 2.998 & 0.5664 & 0.59 \\
90 & 4.442 & 0.964
\end{tabular}

As seen in table 1, the compressibility factor obtained by iteration method obtained is almost equal to the data from software and different temperature compression factor varies widely. The higher the temperature, the smaller the compression factor, gas compressibility factor is greater than 0.5 . The maximum relative error delta is $15.19 \%$, it happens when the temperature is $90^{\circ} \mathrm{C}$. In order to clearly see the iterative calculation accuracy to solve mixed refrigerant compressibility factor, so convert the data to figure. The compressibility factor from different method is shown as figure 2 .

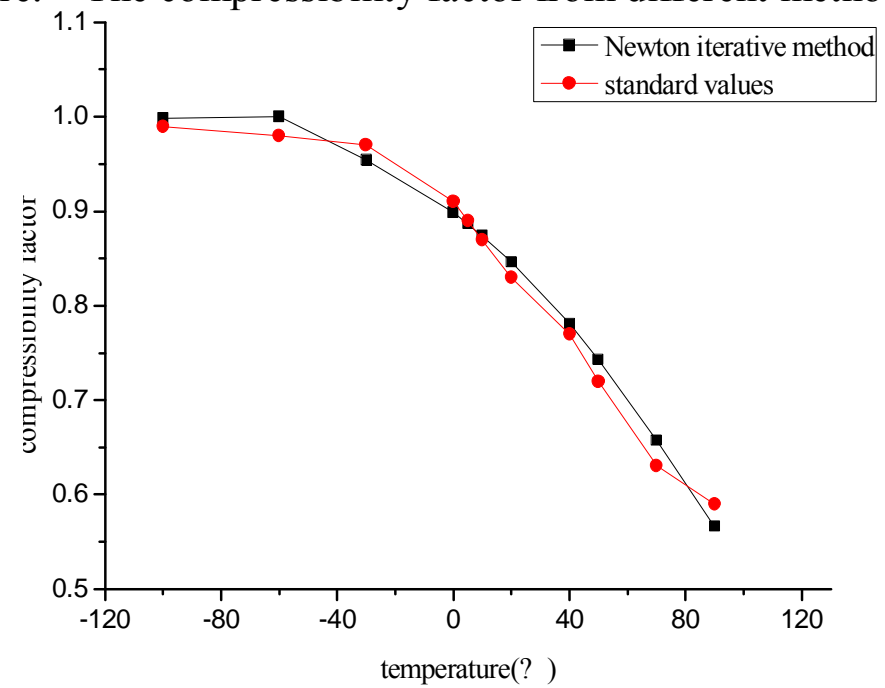

Figure 2 R22 compressibility factor curve

It can be seen from figure 2, the general trend of compressibility factor calculated by Newton iterative method is almost same to the standard values of compressibility factor. The compressibility factor decreases with the increase of temperature, it ranges from 0.55 to 1 . The error is less than $5 \%$, so the solving precision of this program meet the requirements.

\section{Compressibility factor of $\mathrm{CO}_{2}$}

Although R22 refrigerant has been widely used in many refrigeration systems, R22 is about to be eliminated.However, $\mathrm{CO}_{2}$ which is a natural refrigerant, has received widely attention, the value of $\mathrm{CO}_{2}$ compressibility factor is shown as table 2 . 
Table 2. $\mathrm{CO} 2$ compressibility factor

\begin{tabular}{cccc}
\hline $\mathrm{T}\left({ }^{\circ} \mathrm{C}\right)$ & $\mathrm{P}(\mathrm{kpa})$ & Calculation value & Standard value \\
\hline 220 & 599.13 & 0.9092 & 0.91 \\
230 & 892.91 & 0.8794 & 0.88 \\
240 & 1282.5 & 0.8444 & 0.84 \\
250 & 1785 & 0.8039 & 0.81 \\
260 & 2418.8 & 0.7573 & 0.77 \\
270 & 3203.3 & 0.7041 & 0.72 \\
280 & 4160.7 & 0.6427 & 0.64 \\
290 & 5317.7 & 0.5709 & 0.58 \\
300 & 6713.1 & 0.488 & 0.48 \\
\hline
\end{tabular}

Convert the table into figure so as to see clearly, the values of different method is shown as figure 3.

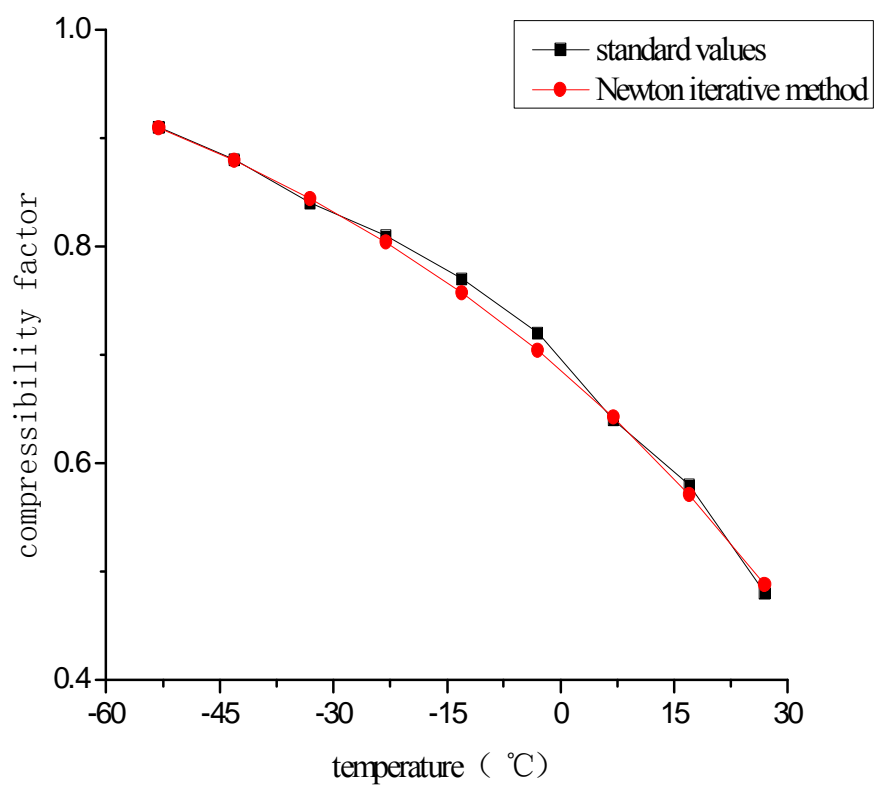

Figure $3 \mathrm{CO} 2$ compressibility factor curve

It can be seen from figure 3 , the values of $\mathrm{CO}_{2}$ compressibility factor is almost same to the standard values. The error of most points is less than $0.1 \%$ and the maximum of error is only $2.2 \%$, so the obtained compressibility factor is more accurate than $\mathrm{R} 22$. The trend of $\mathrm{CO}_{2}$ compressibility factor is same to R22. However, as the two refrigerants has different critical temperature, so the variation sections of temperature is different. From the perspective of the value of obtained compressibility factor, it decreases with the increase of temperature, ranging from 0.48 to 0.91 .

\section{The compressibility factor of mixed refrigerant}

This article mainly solve compressibility factor of R14/R23/R22 in auto-cascade refrigeration system. First of all, the refrigerant R22, R23, R14 is filled in the system to conduct experiment with proportion of 4:3:3. Appropriate illing various refrigerants according to the experiment parameters such as evaporation temperature. After the system runs stable, choose several groups with lower evaporation temperature and record intake and exhaust temperature pressure and temperature. when 
the system runs stable and the evaporation temperature is almost constant, the proportion of R22, $\mathrm{R} 23$, R14 is 4:3.2:2.8. Evaporation temperature changes correspond to temperature is shown as figure 4.



Figure 4 Evaporation temperature changes with time

Based on the program, updated critical pressure, critical temperature and so on. Further more, plug the measured temperature and pressure into program, to achieve compressibility factor as figure5.

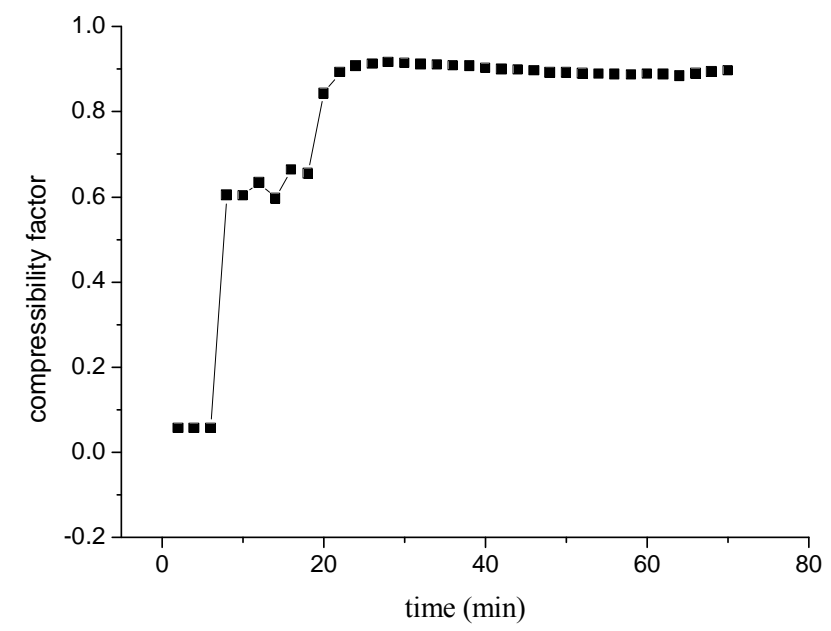

Figure 5 Compressibility factor changes with time

It can be seen from the figure 3, the evaporation temperature decreases over time, after 30 minutes the evaporation temperature is almost constant at about $-90^{\circ} \mathrm{C}$. As shown in figure 4 , the compressibility widely change in 25 minutes, then after 33 minutes, it floats around in 0.9 . It shows that the compressibility factor changes under different states, because the compressibility is different because of the different pressure and temperature. After the system runs stable, the compressibility remains the same. We can come to the conclusion that after the system runs stable, the refrigerator flow in rules and refrigerant composition and gas liquid composition remains unchanged. therefore the compressibility factor remains the same. At last, the compressibility factor of mixed refrigerators is 0.889 when the system reach equilibrium states.

However, the compressibility factor of this system is 0.96 when the ratio of mixed refrigerants (R22/R23/R14) is 4:3.2:2.8. So we can get the conclusion that the component proportion of mixed refrigerants in compressor is unequal to the value of filling. So the component of mixed refrigerants in different device can't calculated with the filling value. The content of gas and liquid refrigerants can calculated with the compressibility factor. 


\section{CONCLUSIONS}

It is an effective and accurate solving method to get compressibility factor $\mathrm{Z}$ that iterative calculate with RKS equation. This method has advantages of simple, convenient and high precision. Compressibility factor can be obtained through software according to actual measured parameters such as temperature and pressure, and then explore the thermal physical properties of mixed working medium, then push the study of theory of the mixed refrigerator. The program is not only suitable for single working medium and it can be applied to mixed working medium cascade refrigeration system. The ratio of refrigerator can be optimized according to the relationship between the compression factor and the evaporating temperature, so as to achieve the purpose of reducing the evaporation temperature and improving the COP.

\section{Acknowledgements}

Heilongjiang Province Natural Science Fund Project: NumberE201231

Heilongjiang Province Graduate Innovation Fund Project: NumberYJSCX2013-240HSD

\section{REFERENCES}

[1]Liu Weidang. Study and development of auto-cascade refrigeration equipment with ternary zeotropic mixture refrigerant[D]. Harbin university of commerce, 2012.

[2]Wang Dongyu. Application of R-K-S state equation in the calculation of Compressibility factor[J]. Journal of Chemical Industry and Engineering, 2001,22(5):32-35.

[3]Mai Yaodi. Determination method of gas compression factor in engineering design.[J]. Guangdong petrol chemical design institude.2006. 16(1):17-18.

[4]Yuan Weimin. numerical methods of using BWRS equation of stete in calculating compressibility factor of natural gas [J]. pipeline technique and equipment, 2009(3):14-16.

[5]Zhou Along. A Thesis Submitted in Partial Fulfillment of the Requirements for the Degree of Master of Engineering [D]. Huazhong University of Science \& Technology dissertation for the Degree of Master[D],2013.

[6]Camporese. R, Bigolaro. G,Rebllato. L. Calculation of thermodynamic properties ofrefrigerants by RKS equation of state. International Journal of Refrigerant, 1985(8):147 151 